\title{
Some Inferences on the Levy distribution
}

\author{
M. Ahsanullah* \\ Rider University, Lawrenceville, New Jersey,USA \\ Valery B. Nevzorov** \\ St. Petersburg State University, St. Petersburg, Russia \\ Received 3 March 2014 \\ Accepted 16 July 2014
}

\begin{abstract}
Some distributional properties of Levy distribution are presented. Limiting distributions of the extreme order statistics are given. Based on the distributional properties a characterization of the Levy distribution is shown.
\end{abstract}

\section{Introduction}

The Levy distribution named after Paul Levy is one of the three stable distributions whose probability density function (pdf) can be expressed in a simple closed form. This distribution was introduced by Paul Levy in1925 during his investigations of the behavior of independent random variables. A random variable is said to have stable distribution if it has the property that a linear combination of two independent copies of the random variable has the same distribution up to a location and scale parameter (see, for example, Zolotarev (1986)) . Stable distributions have been proposed as models in many types of physical and economic problems. The pdf $\mathrm{f}(\mathrm{x})$ of the standard Levy distribution is given by

$$
f(x)=\sqrt{\frac{1}{2 \pi}} \frac{e^{-\frac{1}{2 x}}}{x^{3 / 2}}, \mathrm{x}>0 .
$$

We say that a random variable $X$ has the $\operatorname{Lev}(0,1)$ distribution if its pdf has the form given in (1.1).The pdf $f_{\mu, \sigma}(x)$ of the Levy distribution $\operatorname{Lev}(\mu, \sigma)$ with location parameter $\mu$ and scale parameter $\sigma$ is given by

$$
f(x)=\sqrt{\frac{\sigma}{2 \pi}} \frac{e^{-\frac{\sigma}{2(x-\mu)}}}{(x-\mu)^{3 / 2}}, x>\mu ;-\infty<\mu<\infty, \sigma>0 .
$$

\footnotetext{
*The work for first author was supported by a summer 2014 research grant from Rider University. ** The work of the second author was supported by St.-Petersburg state university ( grant N 6.38.672.2013)
} 
The corresponding cumulative distribution function (cdf) of distribution $\operatorname{Lev}(\mu, \sigma)$ is given by

$$
F_{\mu, \sigma}(x)=2\left(1-\Phi\left(\sqrt{\frac{\sigma}{2(x-\mu)}}\right)\right) \quad, \mathrm{x}>\mu
$$

where $\Phi$ is the cdf of the standard normal distribution.

The pdf $f_{\mu, \sigma}(x)$ is increasing on $(\mu, \mu+\sigma / 3)$ and decreasing on $(\mu+\sigma / 3, \infty)$. This pdf has a long tail with infinite positive moments. The Levy distributions are sometimes used in financial engineering to model price changes because the distribution can take into account the leptokurtosis ('fat' tails) that usually can occur in price changes on financial markets. The Levy distribution with $\mu=0$ is a special case of the inverted gamma distribution with the shape parameter $1 / 2$ and the scale parameter $2 / \sigma$. Montroll and Shlesinger (1983) and Jurlewicz and Weron (1993) have used Levy distribution in physics. In this paper we will discuss some distributional properties of the Levy distribution. The limiting distributions of the extreme and intermediate order statistics are presented. A characterizations of the standard Levy distribution is given.

\section{Main Results}

If the random variable $X$ has the Lev $(\mu, \sigma)$ distribution, then $Y=c X+d$ has $\operatorname{Lev}(c \mu+\mathrm{d}, c \sigma)$. We can write the cdf of the standard Levy distribution as

$$
F(x)=\int_{0}^{x} \sqrt{\frac{1}{2 \pi}} \frac{e^{-\frac{1}{2 u}}}{u^{3 / 2}} d u=2 \int_{1 / \sqrt{x}}^{\infty} \sqrt{\frac{1}{2 \pi}} e^{-\frac{t^{2}}{2}} d t=2\left(1-\Phi\left(\frac{1}{\sqrt{x}}\right)\right), \mathrm{x}>0
$$

where $\Phi$ is the cdf of the standard normal distribution.

The pdfs of $\operatorname{Lev}(0,1), \operatorname{Lev}(0,2)$ and $\operatorname{Lev}(0,4)$ are given in Figure 2.1. 


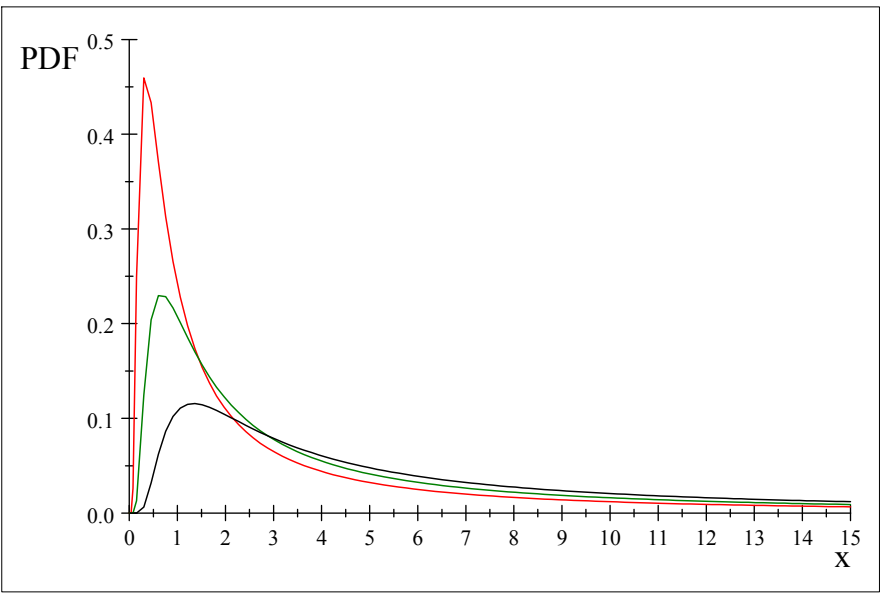

Fig. 2.1. LEV(0,1)-Red, Lev(0,2)-Green, Lev $(0,4)-B l a c k$

The $\operatorname{Lev}(0,1)$ is a unimodal distribution with mode at $x=1 / 3$ and median at $x=2.1081$.

The points of inflection of $\operatorname{Lev}(0,1)$ are at $\frac{1}{2}+\frac{\sqrt{10}}{15}$ and at $\frac{1}{2}-\frac{\sqrt{10}}{15}$.

The following table gives the percentile points of the standard Levy distribution.

\begin{tabular}{|l|l|}
\hline $\mathrm{p}$ & Percentile points \\
\hline 0.1 & 0.3696 \\
\hline 0.2 & 0.6089 \\
\hline 0.3 & 0.9309 \\
\hline 0.4 & 1.4118 \\
\hline 0.5 & 2.1981 \\
\hline 0.6 & 3.6304 \\
\hline 0.7 & 8.7353 \\
\hline 0.8 & 15.5800 \\
\hline 0.9 & 63.328 \\
\hline
\end{tabular}

The characteristic function $\phi_{\mu, \sigma}(t)$ of the random variable $\mathrm{X}$ having $\operatorname{Lev}(\mu, \sigma)$ is given by

$$
\phi_{\mu, \sigma}(t)=\int_{\mu}^{\infty} e^{i t x} \sqrt{\frac{\sigma}{2 \pi}} \frac{e^{-\frac{\sigma}{2(x-\mu)}}}{(x-\mu)^{3 / 2}} d x=e^{i \mu t-\sqrt{-2 i \sigma}} .
$$


The Levy distribution has a heavy tail. For large values of $x$ for $\operatorname{Lev}(0,1)$,

$$
f(x) \sim \sqrt{\frac{1}{2 \pi}} \frac{1}{x^{3 / 2}}, \text { as } x \rightarrow \infty .
$$

Lemma 2.1. If $X$ has the $\operatorname{Lev}(0,1)$ distribution, then $Y=1 / X$ follows the Gamma-distribution with the shape parameter $1 / 2$ and the scale parameter 2.

Lemma 2.2. If $X$ is $N(0,1)$, then $1 / X^{2}$ has the Lev $(0,1)$ distribution.

Lemma 2.3. If $X$ has $\operatorname{Lev}(0,1)$, then $X^{-1 / 2}$ has the folded normal $N(0,1)$ distribution.

Lemma 2.4. If $Z$ has the standard normal $N(0,1)$ distribution, then $X=1 / Z^{2}$ has the Lev $(0,1)$ distribution.

Proof. It is evident, that

$$
P\left(\frac{1}{Z^{2}} \leq u\right)=P\left(Z^{2} \geq \frac{1}{u}\right)=2 P\left(Z \geq \frac{1}{\sqrt{u}}\right)=2(1-\Phi(1 / \sqrt{u})), u>0
$$

which is the cdf of $\operatorname{Lev}(0,1)$.

Lemma 2.5. If $X$ and $Y$ are independently distributed as $\operatorname{LEV}\left(0, \sigma_{1}\right)$ and $\operatorname{Lev}\left(0 . \sigma_{2}\right)$, respectively, then

$$
P(Y<X)=\frac{1}{\pi} \sin ^{-1}\left(\left(\frac{\sigma_{1}}{\sigma_{1}+\sigma_{2}}\right)^{1 / 2}\right)
$$

Proof. We have

$$
P(Y<X)=\int_{0}^{\infty} 2\left(1-\Phi\left(\frac{\sigma_{2}}{\sqrt{x}}\right)\right) \sqrt{\frac{\sigma_{1}}{2 \pi}} \frac{e^{-\frac{\sigma_{1}}{2 x}}}{x^{3 / 2}} d x=\frac{2}{\pi} \sin ^{-1}\left(\left(\frac{\sigma_{1}}{\sigma_{1}+\sigma_{2}}\right)^{1 / 2}\right) .
$$

If $\sigma_{1}=\sigma_{2}$, then $\left.P(Y<X)\right)=0.5$.

It follows from $(2.1)$ that if $X$ has the $\operatorname{Lev}(0, \sigma)$ distribution, then the survival function $\bar{F}(x)=1-F(x)$ is given by

$$
\bar{F}(x)=P(X \geq x)=2 \Phi\left(\sqrt{\frac{\sigma}{x}}\right)-1, x \geq 0
$$

The graphs of the survival functions of Levy distributions are given in figure 2.2. 


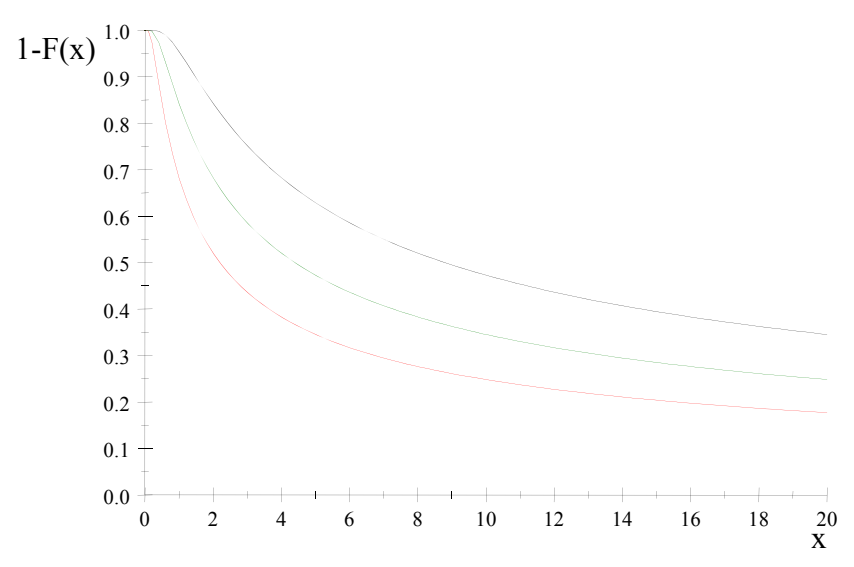

Fig.2.2. 1-F(x): Lev (0,1)-Red,Lev(0,2)-Blue, Lev(0,4)-Black

The survival function is a decreasing function.

The positive moments of the Levy distribution do not exist. But the negative moments exist. If $X$ has the $\operatorname{Lev}(0,1)$ distribution, then

$$
E\left(X^{-k}\right)=\int_{0}^{\infty} x^{-k} \sqrt{\frac{1}{2 \pi}} \frac{e^{-\frac{1}{2 x}}}{x^{3 / 2}} d x=\prod_{i=1}^{k}(2 i-1)
$$

for any positive integer $\mathrm{k}=1,2, \ldots$

We have

$$
\lim _{n \rightarrow \infty} \frac{F^{-1}\left(1-\frac{1}{n}\right)-F^{-1}\left(1-\frac{2}{n}\right)}{F^{-1}\left(1-\frac{2}{n}\right)-F^{-1}\left(1-\frac{4}{n}\right)}=4
$$

Thus, using Theorem 2.1.5 of Ahsanullah and Nevzorov (2001), one gets that with proper normalizing constants

$$
P\left(X_{n, n} \leq a_{n}+b_{n} x\right) \rightarrow e^{-x^{-1 / 2}}
$$

for all $x \geq 0$, where $X_{n, n}=\max \left(X_{1}, X_{2}, \ldots, X_{n}\right)$ and the random variables $X_{i}, i=1,2, \ldots$ are independent and identically distributed. 
Note that here $a_{n}=0$ and $\mathrm{b}_{\mathrm{n}}=F^{-1}\left(1-\frac{1}{n}\right) \sim \frac{2 n^{2}}{\pi}, n \rightarrow \infty$.

We have also that

$$
\lim _{n \rightarrow \infty} \frac{F^{-1}\left(\frac{1}{n}\right)-F^{-1}\left(\frac{2}{n}\right)}{F^{-1}\left(\frac{2}{n}\right)-F^{-1}\left(\frac{4}{n}\right)}=1
$$

Thus by Theorem 2.1.5 of Ahsanullah and Nevzorov (2001) it follows that

$$
P\left(X_{1, n} \leq c_{n}+d_{n} x\right) \rightarrow 1-\exp \left(-e^{x}\right),-\infty<x<\infty, \text { as } \mathrm{n} \rightarrow \infty,
$$

where $X_{1, n}=\min \left(X_{1}, X_{2}, \ldots, X_{n}\right)$ and the random variables $X_{1}, X_{2}, \ldots, X_{n}$ are independent and identically distributed. The normalizing constants are given here as

$$
c_{n}=F^{-1}\left(\frac{1}{n}\right) \text { and } \mathrm{d}_{\mathrm{n}}=F^{-1}\left(\frac{1}{n}\right)-F^{-1}\left(\frac{1}{n e}\right) .
$$

\section{A Characterization.}

Theorem 3.1. Let $X_{1}, X_{2}, X_{3}$ be independent identically distributed absolutely continuous random variables with cdf $F(x)$ and $p d f f(x)$. We assume $F(0)=0$ and $F(x)>0$ for all $x>0$. Then $X_{1}$ and $\left(X_{2}+X_{3}\right) / 4$ are identically distributed if and only if $F(x)$ has the Lev $(0, \sigma)$ distribution.

Proof.

Necessity.

Suppose that $X_{1}, X_{2}$ and $X_{3}$ have the Levy distribution with pdf $f(x)$ having the form

$$
f(x)=\sqrt{\frac{\sigma}{2 \pi}} \frac{e^{-\frac{\sigma}{2 x}}}{x^{3 / 2}}, x \geq 0, \sigma>0
$$

Then the characteristic function of $X_{I}$ is given as

$$
\phi(t)=e^{-\sqrt{-2 i \sigma t}}
$$

The characteristic function of $\left(X_{2}+X_{3}\right) / 4$ is as follows:

$$
\left(\exp ^{-\sqrt{2 i \sigma t / 4}}\right)^{2}=e^{-\sqrt{-2 i \sigma t}}=\phi(t)
$$

\section{Sufficiency.}

Suppose that $X_{1}$ and $\left(X_{2}+X_{3}\right) / 4$ are identically distributed. Then we have 


$$
\phi(t)=\left(\phi\left(t / 2^{2}\right)\right)^{2}=\ldots=\left(\left(\phi\left(t / 2^{2 n}\right)\right)^{2^{n}}, \mathrm{n}=1,2, \ldots\right.
$$

Denote $\psi(t)=(\ln \phi(t))^{2}$. Then we have that

$$
\psi(t)=2^{2}(\psi(t / 4)) . .=2^{2 n} \psi\left(t / 2^{2 n}\right) .
$$

The solution of the above equation is

$$
\Psi(t)=c t
$$

where $c$ is a constant, and hence

$$
\phi(t)=e^{-\sqrt{c t}}
$$

Using the condition that $\phi(-t)=\bar{\phi}(t)$, where $\bar{\phi}(t)$ is the complex conjugate of $\varphi(t)$, one gets that $c$ can be taken as $c=-2 i \sigma$, where $i^{2}=-1$ and $\sigma>0$ is a constant.

The proof is complete.

\section{References}

[1] Ahsanullah,M. and Nevzorov,V.B. Ordered Random Variables. Nova Science Publishers, New York, 2001.

[2] Jurlewicz, A. and Weron,K. A relationship between asymmetric Levy-stable distribution and dialectic susceptibility. Journal of Statistical Physics, 73, N1-2, pp.69-81,1993.

[3] Montroll, E. W. and Shlesinger, M. F. Maximum entropy formalizm, fractals, scaling phenomena, and 1/f noise: a tale of tails. Journal of Statistical Physics, 32, N2, pp.209-230, 1983.

[4] O'Reilly ,F.J. and Rueda,H. A note on the fit for the Levy distribution. Commun. Stat.-Theor. Meth., 27, N7, pp.1811-1821, 1998.

[5] Zolotarev,V.M. One dimensional stable distributions, vol.65 (Translation of Mathematical Monograph), American Mathematical Society, 246p., 1986 (translation from the original 1983 Russian edition). 